\title{
Space-Time Cube and Mixed Reality - New Approaches to Support Understanding Historical Landscape Changes
}

\author{
Mathias Jahnke $^{\mathrm{a}, *}$, Edyta P. Bogucka ${ }^{\mathrm{a}}$, Maria Turchenko ${ }^{\mathrm{a}}$ \\ ${ }^{a}$ Technical University of Munich, mathias.jahnke@tum.de,e.p.bogucka@tum.de, maria.turchenko@gmail.com \\ * Corresponding author
}

\begin{abstract}
Mixed reality is a rather new technology but came to its nowadays success through the availability of devices like Microsoft HoloLens which easily support the users and developers to use such devices. Therefore, visualization specialists like cartographers paid attention due to interaction possibilities such devices provide. In particular, to utilize the huge amount of opportunities such device gave. The applicability within the cartographic domain needs to be further investigated.
\end{abstract}

The main goal of this contribution is to evaluate the applicability of a mixed reality device in the domain of spatiotemporal representations on the example of the space-time cube to show cultural landscape changes. The hologram of the space-time cube provides the changes of the Royal Castle in Warsaw and their surrounding elements. The hologram therefore incorporated the different buildings of the castle, space-time prisms and space-time links to connect building elements over the years. The visual variables colour hue, colour value and transparency are mainly used to feature distinguishable space-time prisms and to show the space-time links. Different colour schemes are developed which features the characteristics of a mixed reality device. The possibilities of input actions are ranging from gaze/head movement, to gesture and voice.

The usability evaluation of the mixed reality hologram showed the overall comfort of interactions, perception of the visual components of the space-time cube and determines advantageous features and limitations of the technology. Most of the found limitations are connected to current devices, like e.g. resolution or field of view. An important aspect which came out is, that the experience the user has which such devices/technology plays an important role in successfully use and knowledge discovery from such applications.

Keywords: mixed reality, virtual hologram, space-time cube, landscape changes

\section{Introduction}

The variety of geovisualizations and visual analytical tools is utilizing computer displays as primary devices for visual perception and interaction with geographical information. Depiction of spatio-temporal data is a challenging task and plays an important role in visual analytics and knowledge discovery processes (Bruggmann and Fabrikant 2016). Typical spatio-temporal application scenarios are on the one hand the visualization and analysis of trajectories of moving objects to understand the behaviour of car drivers, and, on the other hand, the representation of long-lasting landscape changes covering multiple centuries (Bogucka and Jahnke 2018). These scenarios can be visualized using maps, scatterplot matrices, parallel coordinate plots or graphs (Kehrer and Hauser 2013). As the landscape objects such as buildings own a spatial location and lifespan (creation - destruction), mapping those attributes can provide new insights into the development of a city or the landscape in general. This contribution focuses on the spatio-temporal representation of landscape continuum and utilizes well-established spatio-temporal visualization technique from visual analytics together with the recent advances in mixed reality domain.

\subsection{Space-Time Cube and their elements}

A common approach to visualize spatio-temporal data is the space-time cube. This visualization technique originates from the humanities research investigating human movements in space and time (Hägerstrand 1970, Kveladze et al. 2013) and can be adapted to the landscape analysis. The landscape consists of multiple visible features, which are subject to either long-lasting changes, e.g landforms or to relatively rapid modifications, e.g. buildings and vegetation. Within the cube features are placed vertically along the z-axis (time-axis) and horizontally on the $\mathrm{x}-$, $\mathrm{y}$-planes (space-time prisms). As some of the landscape changes are observable in the longtime perspective, the time axis of the space-time cube covers many centuries and indicates two units of measurement simultaneously: 1) the time as a chronological order of the positions of the space-time prisms and 2) the objects dimensions as a continuous scale (Bogucka and Jahnke 2018). If the study area contains many changing objects, they can be grouped into fewer time prisms, e.g one prism for each century, to avoid visual cluttering. Identification of expansion, destruction and rebuilding of single objects as well as tracking the spatial dimensions of those changes through time might be 
difficult (Bogucka and Jahnke 2017). Therefore, the original idea of space-time paths was replaced by spacetime links - vertical lines which connect main corners of the same objects between time prisms.

\subsection{Mixed Reality Visualization}

Mixed Reality (MR) and Augmented Reality (AR) concepts are present in a wide range of applications and visualizations. Milgram and Kishino (1994) place MR within their Reality-Virtuality continuum as related to the real environment on the one side of the continuum and the virtual environment on the opposite side (Figure 1). Therefore, mixed reality implies real world and virtual digital objects co-existing and influencing each other. Real-world objects are accompanied by virtual overlays and virtual environments are supplemented by real world objects. In practical applications, MR combines the real world with three-dimensional virtual holograms. Replacing computer displays with holograms is a step towards the captivating perception and the higher degree of interactivity freedom with the visualization. Therefore, it is crucial to investigate to what degree and if the existing web-based visualization approaches can be implemented in the MR environment.

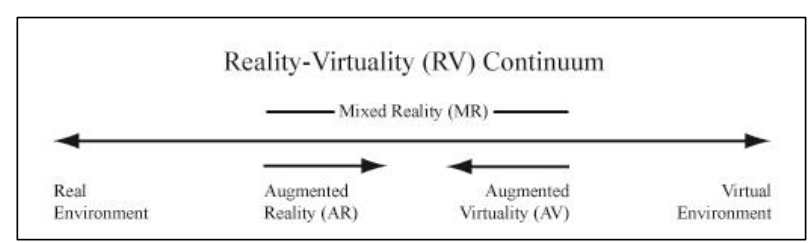

Figure 1. Mixed Reality within the Reality-Virtuality Continuum (adapted from Milgram and Kishino 1994).

Due to its promising natural ways of interaction with data and giving the user a more immersive feeling, MR could be of a potential use in the fields of cartography and visual analytics. This research attempts to transfer the existing web-based representation of the space-time cube (Figure 2) into a MR space-time cube and point out possibilities and limitations of this updated tool. The remainder of the paper is organized into three sections. The first section introduces the case study of the historical landscape and proposes the steps towards bringing space-time cube elements and interactivities in MR. The second section reports on the implementation workflow, while the third one presents the results of the user study. The last section summarizes the results of this work and draws conclusions for future development.

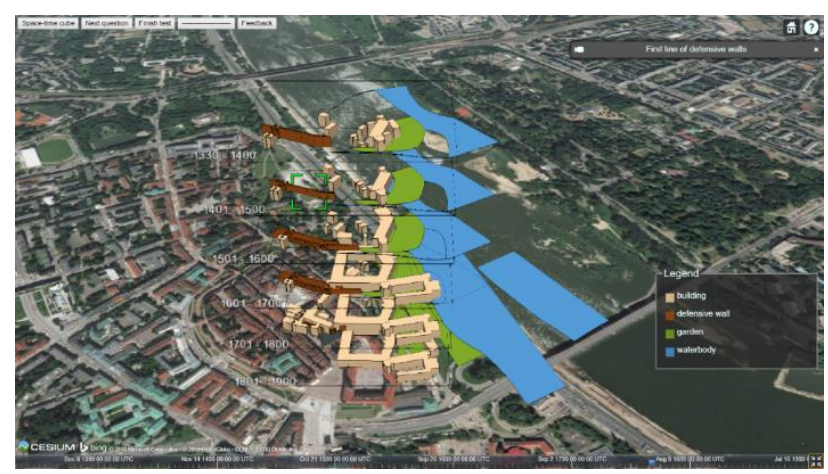

Figure 2. Space-time cube with landscape elements implemented in web-based technologies (Bogucka and Jahnke 2018).

\section{The case study}

The practical implementation of the space-time cube is based on the case study of the Royal Castle in Warsaw, Poland, registered in 1980 as a part of Warsaw's UNESCO World Heritage Site. The study area includes different spatial elements: the castle itself, adjoining gardens and the outbuildings - the Tin-Roofed Palace and the Kubicki Arcades (Figure 3). In its 600-years history, the castle underwent significant changes, such as large-scale expansions, partial destructions and annihilation during the World War II. Since 1971, the residence is in the ongoing restoration process. Therefore, the holographic visualization can help to tell the unique story of the castle in an immersive and engaging way. Future visitors of the castle could explore not only different historical development phases, but also possible effects of reconstruction works.

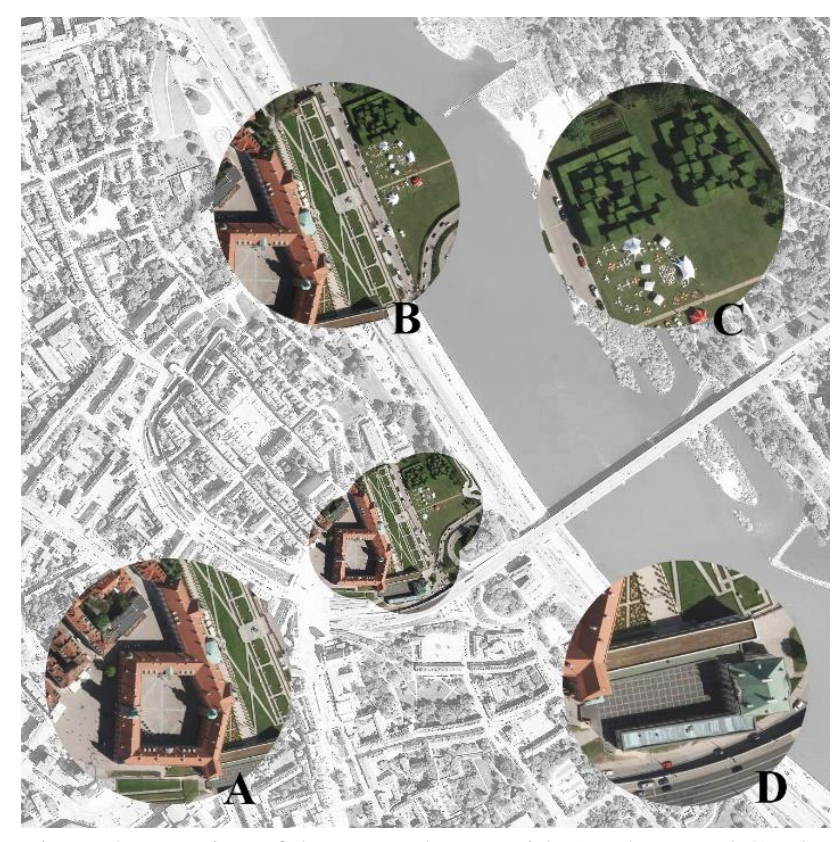

Figure 3. Location of the research area with A - the Royal Castle, B - the Upper Garden and the Kubicki Arcades, C - the Lower Garden and D - the Tin-Roofed Palace. Background map source: Office of Surveying and Cadastre, Warsaw: www.mapa.um.warszawa.pl. 
Based on these pre-requisites, a holographic space-time cube for the castle may consist of the following basic forms and elements:

- the landscape is simplified to show only buildings and water bodies in the standard of City GML LoD1 (Kolbe et. al 2005),

- $\quad$ historical buildings are represented as 3D-cuboids. Colour hue is utilized to classify the buildings based on the century they originate from. Different heights and widths for the buildings (size) are derived from the buildings' footprints through their extrusion,

- $\quad$ similarly to buildings, water bodies are implemented as extruded 3D-volumes and symbolized with the blue colour,

- space-time links utilize transparency to connect buildings' locations through the temporal dimension of the cube,

- time prisms are represented as semi-transparent ground slices and put at the base of the landscape objects for each time subinterval,

- each prism displays landscape changes grouped in 100-year intervals, starting from the year 1400 up to 2000. The final cube consists then from 7 space-time prisms instead of 600 .

\subsection{Implementation of the hologram}

The development of the hologram started with temporally labelled footprints of the case study area (Figure 4). These footprints were extruded using ESRI ArcScene (10.5) and placed onto the right time prism. Afterwards the model was re-meshed with Blender (version 2.79) and converted to match the format of Unity (version 2018.3). Unity and C\# programming language served as the basis environment for implementing different interactions such as showing metainformation, rotation, panning and zooming in the virtual model. Finally, Visual Studio 2017 was used to transfer the implemented application to the Microsoft HoloLens device (first generation).

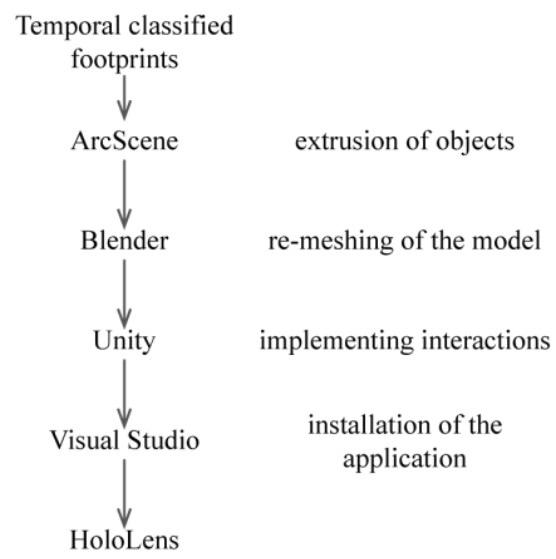

Figure 4. The workflow of implementing the hologram in the HoloLens.
The developed virtual hologram integrates spatio-temporal elements suitable for this thematic domain (historical objects, time prisms, space-time links) with cartographic principles and map elements (Figures 4-5). Special attention was paid to visual variables of colour hue, colour value, size and transparency. While the historical objects (buildings and water bodies) were mainly designed using colour hue to distinguish between the different time prisms, colour value was used to highlight those elements. Transparency was applied to visualize space-time links and the ground layer of each time prism. As MR applications are characterized by the non-static background, the overall transparency of the visualization varies for different colours and textures. Therefore, colour schemes had to be defined very carefully and transparency as a visual variable was used with caution. The visual variables of colour hue and colour value (based on recommendations from ColorBrewer $2^{1}$ ) were adjusted for the HoloLens display rendering.

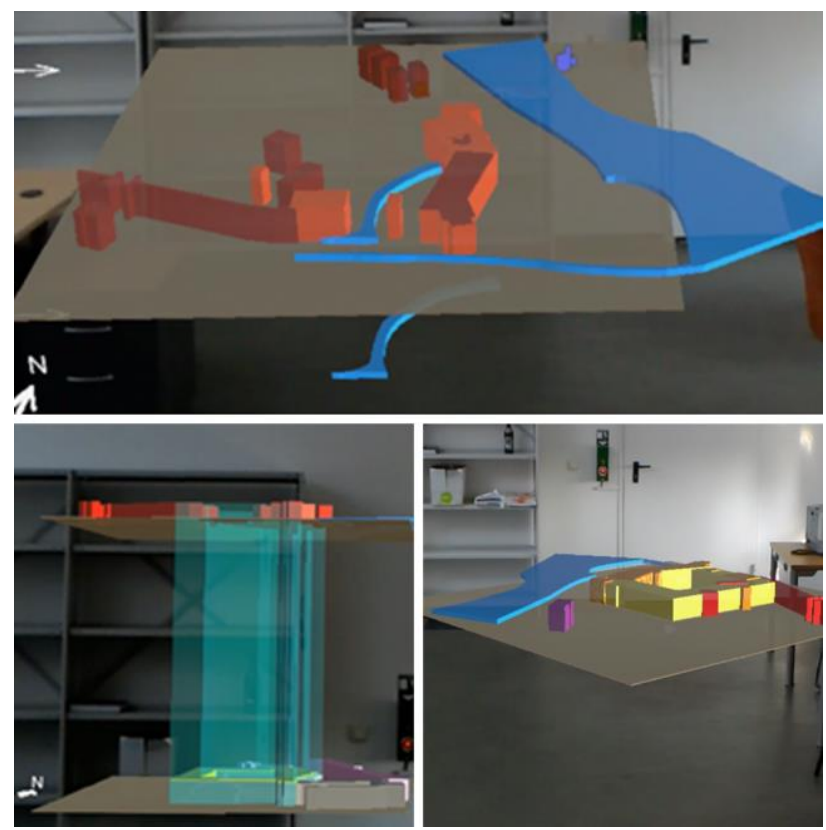

Figure 4. Basic forms of the space-time cube in MR. Upper part of the figure presents historical buildings and water bodies; in the lower left corner space-time links are visible between two spacetime prisms; in the lower-right corner single prism is visible.

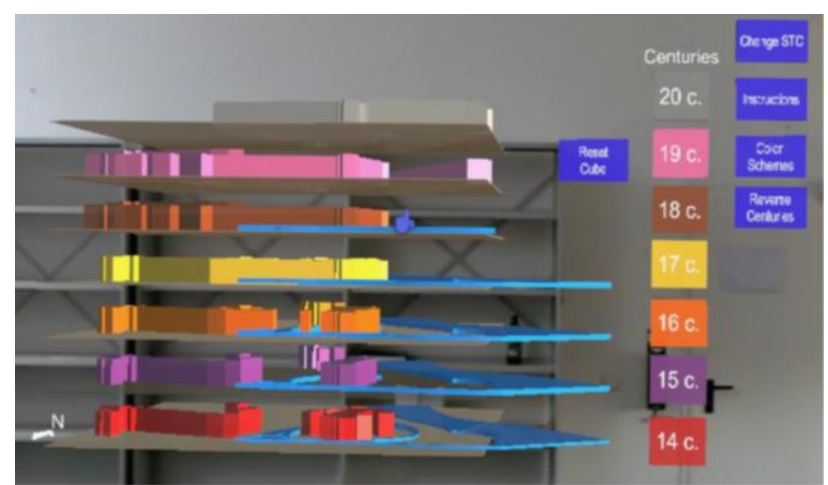

\footnotetext{
${ }^{1} \mathrm{http} / / /$ colorbrewer2.org
} 
Figure 5. The main interface of the space-time cube in MR with basic map elements - figure, legend and north arrow.

Interactivity of the developed holographic application attempts to make use of novel interaction possibilities of the mixed reality, such as gesture, voice input and gaze input (Table 1). Figure 6 presents how gaze- and gesturebased interactivities are organized in a visual interface of the hologram.

Table 1: Interactions implemented to use the hologram

\begin{tabular}{|c|c|}
\hline $\begin{array}{l}\text { Interaction } \\
\text { mode }\end{array}$ & Interaction description \\
\hline Gaze & $\begin{array}{l}\text { Highlighting - change of the colour hue, when } \\
\text { the user's gaze intersects the landscape feature's } \\
\text { location in 3D MR space }\end{array}$ \\
\hline \multirow{9}{*}{ Gesture } & $\begin{array}{l}\text { Interactive labelling - air tap on the landscape } \\
\text { feature opens an info window with feature's } \\
\text { name, century of construction and years of } \\
\text { existence }\end{array}$ \\
\hline & $\begin{array}{l}\text { Dragging the info window - moving the } \\
\text { position of the pop-up information by selecting } \\
\text { it and dragging to a preferable place }\end{array}$ \\
\hline & $\begin{array}{l}\text { Space-time linking - toggle visibility of the } \\
\text { space-time links through the dedicated button }\end{array}$ \\
\hline & $\begin{array}{l}\text { Rotation - moving the cube in vertical and } \\
\text { horizontal planes through interactions with the } \\
\text { bounding box }\end{array}$ \\
\hline & $\begin{array}{l}\text { Resetting the view - changing the rotation of the } \\
\text { space-time cube to its initial position by tapping } \\
\text { "Reset" button in the user interface }\end{array}$ \\
\hline & $\begin{array}{l}\text { Scaling the cube - user can select cube's } \\
\text { vertices and move the hand towards the center } \\
\text { of the cube to make the cube smaller and away } \\
\text { from the centre to enlarge the visualization. }\end{array}$ \\
\hline & $\begin{array}{l}\text { Switching between colour schemes - tapping } \\
\text { the "Color Schemes" button visualizes the data } \\
\text { in diverging, qualitative or sequential colour } \\
\text { scheme. }\end{array}$ \\
\hline & $\begin{array}{l}\text { Filtering visualized time subintervals - toggling } \\
\text { the visibility of each separate time subinterval } \\
\text { (ground slice with landscape features) by } \\
\text { tapping correlated legend element in the user } \\
\text { interface. }\end{array}$ \\
\hline & $\begin{array}{l}\text { Re-ordering of the time subintervals along the } \\
\text { time axis - tapping "Reverse Centuries" button } \\
\text { changes the order of the time subintervals } \\
\text { (centuries) from the contemporary landscape } \\
\text { time subinterval on the top of the cube to its } \\
\text { positioning at the bottom. }\end{array}$ \\
\hline \multirow[b]{2}{*}{ Voice } & $\begin{array}{l}\text { Rotation-moving the cube in vertical plane. To } \\
\text { start rotation user should say the voice } \\
\text { command "Vertical rotation", to stop it - "Stop } \\
\text { vertical rotation" }\end{array}$ \\
\hline & $\begin{array}{l}\text { Dragging the hologram - shifting the cube in } \\
\text { any direction. User should say the voice } \\
\text { command "Move Cube" to initiate the } \\
\text { interaction and "Stop moving" to finish the } \\
\text { operation. }\end{array}$ \\
\hline
\end{tabular}

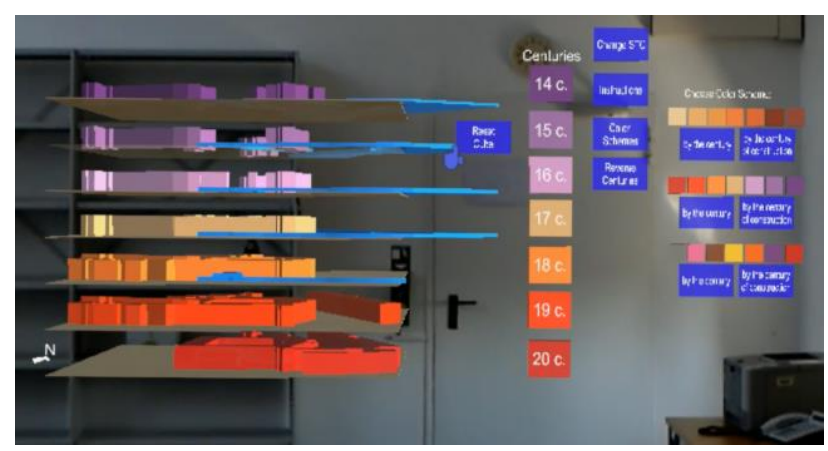

Figure 6. Interface of the holographic space-time cube.
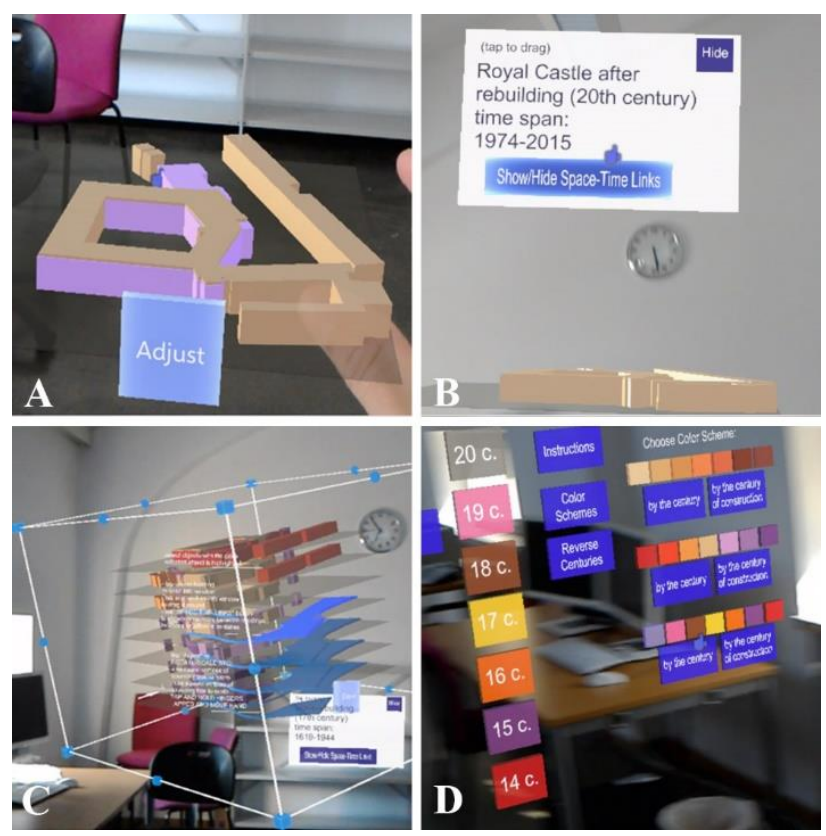

Figure 7. Selected interactions with the holographic cube: A highlighting with the gaze, $\mathrm{B}$ - interactive labelling, $\mathrm{C}$ - rotation with the bounding box, $\mathrm{D}$ - changing colour schemes.

\section{Evaluation of the Hologram}

\subsection{Design of the user study}

To get an initial idea of the usability of the developed hologram, a user study was conducted among 20 (12 males, 8 females) users. The age range of the participant went from 21 years to 41 years whereas $65 \%$ of the participants were between 25 years and 34 years old. The background of the participants was quite divers like e.g. cartography, geoinformatics, computational mechanics and mechanical engineering. The biggest group with $40 \%$ of the participants had a cartographic background.

Two different design versions and ways of interacting (gesture and voice) with the HoloLens were developed. The first design version had $90 \%$ transparency of the ground slices, a sequential colour scheme, categorized by the century, as default. Rotation, scaling panning of the space-time cube were performed through gestures. The second design version had $10 \%$ transparency of the ground 
slices, which made them almost opaque, a qualitative colour scheme, categorized by the century, as a default. Rotation and panning operation was performed by means of voice commands, scaling of the space-time cube was disabled within this scenario.

Two pre-tests specified the final experiments and guaranteed a suitable and feasible procedure. The final user study was divided into three steps, starting with an introduction to the technology followed by a task based test and ended with a questionnaire. Every user was provided with the same amount of information. The time to accomplish each individual test lasts between 30 to 50 minutes.

After a short introduction to the technology and time to learn the interactions with the mixed reality device the participants were asked to find answers to four spatiotemporal tasks:

- What was the most northern building in the 16th century?

- Which water bodies in the 14 th century were located at the same place as the Royal Castle after rebuilding in the 20th century?

- How many buildings were represented in the 18th century?

- Name the oldest buildings existing in the 17th century?

To find the correct answer to those tasks gaze, gesture or voice interactions were used. Every question could be answered by applying a set of intended task to find the correct result. By answering these questions users explored and evaluated several components of the space-time cube design in the mixed reality, among them perception of the cube, overall comfort of interactions, and limitations of the technology.

\subsection{Results of the user study}

Thus, the main finding about interactions with the spacetime cube hologram concerned the previous experience they had with mixed reality devices. The users who are familiar with mixed reality demonstrated faster task performance in comparison to the users without such an experience (Figure 8).

Influence of previous mixed reality experience on the task performance

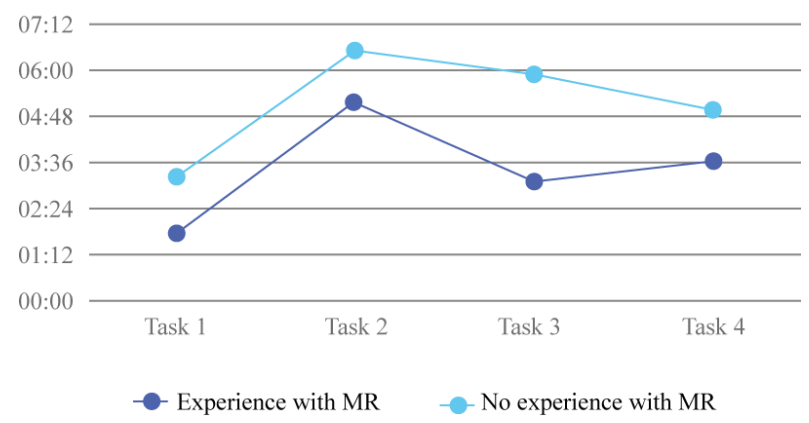

Figure 8: Task/answering performance of user with and without mixed reality experiences
Beyond all participants, displaying meta-information for buildings and filtering centuries are the main interactions used in these scenarios, followed by typical user interactions like rotating and panning (dragging). Interaction tasks like switching the colour scheme or reversing the order of centuries was less used to find correct answers (Figure 9).

Frequency of using interactive features

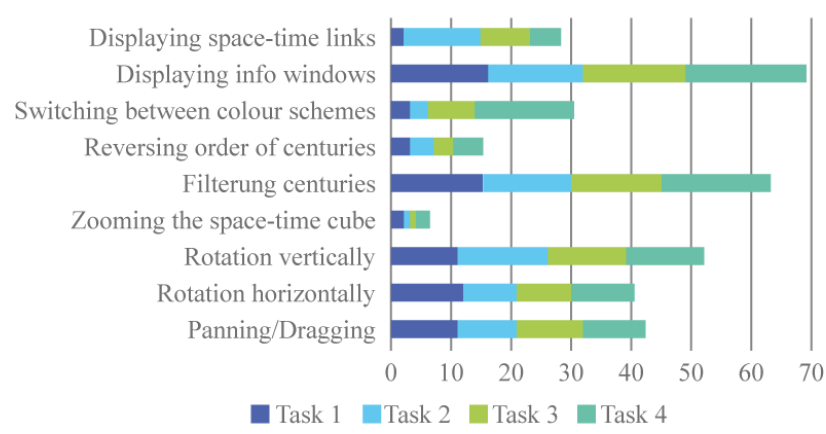

Figure 9: Frequency of interactions used for the different tasks.

The interaction through gaze, gesture and voice gained positive feedback from the users, but it was essential to have enough time to learn the interaction and input methods to get the feeling of immersion with historical data. Interacting with small objects was evaluated most difficult while selecting objects and navigating the hologram was evaluated as medium difficult (Figure 10).

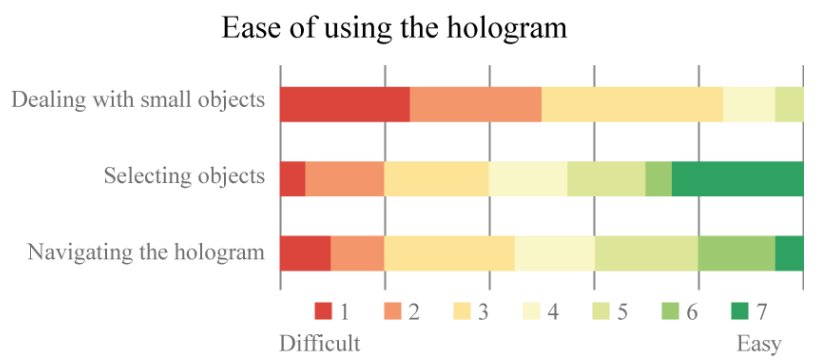

Figure 10: Generalized comfortability of interactions with the hologram

The participants appreciated the novelty of this technology such as natural way of interaction, enjoyable user experience, visual appearance and control through voice (Figure 11); however, they pointed several technical limitations that have negatively influenced their experience. The problems were caused by a limited size of the holographic screen of the headset, limited field of view while working with the hologram as well as time to learn hand gestures and the system reaction time on gestures and voice commands. Additionally, the gaze selection was not working smooth while focusing on the small objects. 


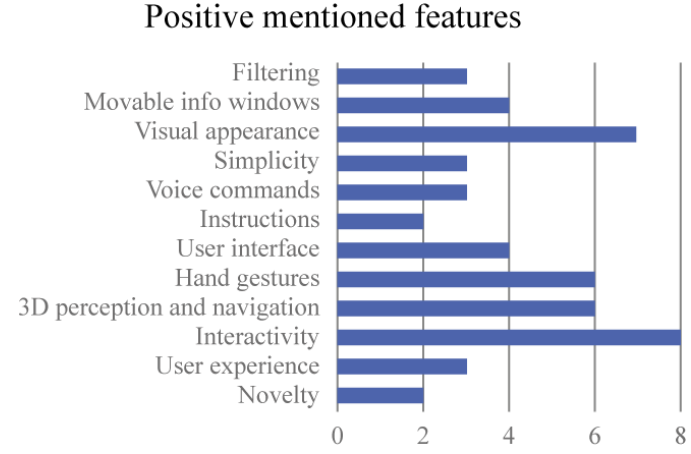

Figure 11: feature which are favourable for a mixed reality application

Analysing the collected video material throughout the user study, showed that in particular those participants with no experiences in mixed reality application have had more difficulties to form gestures which are correctly recognized by the system.

\subsection{Discussion on the results}

Experiences in using mixed reality applications or devices gained in faster task performance compared to those without experiences which corresponds to findings from Yim et al. (2016). Working with the space-time cube (showing information, rotating etc.) helped more to correctly accomplish the tasks than to change the style or design of the visualization (different colour schemes, revers ordering of centuries). The difficulties of selecting relatively small objects is another aspect which turned out. An explanation for this could be on the one hand the relatively low resolution together with detecting the movement detection of the device and on the other hand the relatively flat angle between the objects and the viewing direction of the user. This leads to the conclusion that intuitive zooming should be integrated and that developers must carefully design such applications.

The following advantages of a mixed reality application can be summed up:

- 3D perception and navigation, possibility to move around;

- natural way of interaction through hand gestures and gaze selection;

- interactivity and a wide range of functionalities;

- enjoyable user experience and visual appearance;

- control through voice;

- $\quad$ novelty and the feel of the future technology.

While the limitations and drawbacks can be summarized as follows:

- $\quad$ focusing of the gaze on the small objects;

- $\quad$ system reaction time on hand gestures;

- limited size of the holographic screen of the mixed reality device and limited field of view;

- $\quad$ long response time for the hand detection and voice recognition;

- $\quad$ poor contrast within brightly lighted spaces.

\section{Conclusions}

This contribution aimed at determining how new visualizations approaches such as mixed reality visualizations can influence the visual analytics and knowledge discovery processes as well as what kind of limitations are existent.

Mixed reality device like Microsoft HoloLens are opening new ways of communicating data and interacting with data. Therefore, the space-time cube visualization of the changes of the Royal Castle of Warsaw were transferred from a $3 \mathrm{D}$ browser based visualization to a mixed reality visualization. Colour schemes and design principles needed some adaptation to work in a hologram, colour hue, colour value and transparency were mainly used to convey the desired information but need some adaptation to the light condition of the surrounding. Especially the user test showed that the visualization of a hologram needs to be carefully designed to bring the information to the user and to support knowledge discovery in such scenarios.

The developed space-time cube visualization was evaluated and proven positively if the users have some prior knowledge concerning the technology and its procedures.

\section{Acknowledgements}

The authors would like to thank all participants of the user study for their valuable feedback.

We are grateful to the experts from Royal Castle in Warsaw - Museum, who provided historical data, insight and expertise that greatly assisted the research.

The study was based on source materials available in Poland in the public domain, which meet the criteria for re-use in accordance with the Act of 6 September 2001 on Access to Public Information (Dz. U. of 2014., Pos. 782, as amended).

This work was carried out as a Master's thesis research within the international Master of Science in Cartography program.

\section{References}

Bogucka, E. P. and Jahnke M. (2018). Feasibility of the Space-Time Cube in Temporal Cultural Landscape Visualization. ISPRS International Journal of GeoInformation, 7 (6), 209.

Bogucka, E. P. and Jahnke, M. (2017). Space-Time Cube - A Visualization Tool for Landscape Changes. Kartographische Nachrichten, 66(4), 183-191.

Bruggmann, A., Fabrikant, S.I. (2016). How does GIScience support spatio-temporal information search in the humanities? Spatial Cognition \& Computation, 16(4), 255-271.

Kehrer, J., Hauser, H. (2013). Visualization and Visual Analysis of Multifaceted Scientific Data: A Survey. IEEE Transactions on Visualization and Computer Graphics, 19(3), DOI:10.1109/TVCG.2012.110. 
Kolbe T.H., Gröger G., Plümer L. (2005) CityGML: Interoperable Access to 3D City Models. In: van Oosterom P., Zlatanova S., Fendel E.M. (eds) Geoinformation for Disaster Management. Springer, Berlin, Heidelberg

Kveladze, I.; Kraak, J.-M.; Van Elzakker, C. P. J. M. (2013). A Methodological Framework for Researching the Usability of the Space-Time Cube. The Cartographic Journal, 50, 201-210, DOI: 10.1179/1743277413Y.0000000061

Hägerstrand, T. (1970). What about people in regional science? Papers in Regional Science Association. 24, 721.

Milgram, P. and Kishino, F. (1994). 'A taxonomy of mixed reality visual displays', IECEI Transactions on Information Systems, E77-D, pp. 1321-1329.

Yim, Dianna, Loison, Garance Nicole, Fard, Fatemeh Hendijani, Chan, Edwin, McAllister, Alec, and Maurer, Frank. "Gesture-driven Interactions on a Virtual Hologram in Mixed Reality.” 2016. 